\title{
Multi-knowledge Extraction from Violent Crime Datasets Using Swarm Rough Algorithm
}

\author{
Chao Yang, Hongbo Liu, Yeqing Sun \\ Institute of Environmental Systems Biology \\ Dalian Maritime University \\ Dalian 116026, China \\ yangchaoneu@sina.com, \{lhb,yqsun\}@dlmu.edu.cn
}

\author{
Ajith Abraham* \\ IT For Innovations, VSB - Technical University of Ostrava, \\ Czech Republic \\ *Machine Intelligence Research Labs \\ Scientific Network for Innovation and Research Excellence \\ Auburn, Washington 98071, USA \\ ajith.abraham@ieee.org
}

\begin{abstract}
This paper presents a swarm rough approach to analyze the combination factors of violent crime. The approach discovers the feature combinations in an efficient way to observe the change of rough set positive region as the fuzzy swarm proceed throughout the search space. We evaluated the performance of our approach using the violent factor datasets and the corresponding computational experiments are discussed. Empirical results indicate that our approach is ideal for all the considered problems and the fuzzy swarm optimization technique outperforms dynamic reducts (DR) approache by obtaining multiple reductions for the combination factor datasets.
\end{abstract}

Keywords-Rough sets; Swarm intelligence, Fuzzy swarm optimization; Multi-knowledge extraction; Violent behavior analysis

\section{INTRODUCTION}

Violent crimes take violence behaviors of infringing upon other people to obtain some interest or satisfy a certain desire, including eight categories of violence cases: murder, explosion, poisoning, arson, hijacking, kidnapping, rape and intentional injury [1]. It not only causes severely victims' bodily injury and psychological trauma, but also seriously harms victims' families and public stability. The incidence rate of violent crimes is small, however, its social harmfulness is much severer than other crimes, so to prevent violent crimes are the focus of police work. Therefore, to explore the reasons of violent crime and the search for effective management and educational measures have been the key areas of sociology and criminology research. Currently, in the field of bioinformatics, a large number of genes about criminal factors have been excavated, which have the meaning of the milestone for the key of the detecting crime [2], [3], [4], [5]. At the same time, psychology has also been widely applied to the correlation analysis of crime factors, and it reveals the implicit relation between psychological motives. For example, the personality can be analyzed by the Eysenck Personality Questionnaire (EPQ) [6], [7]. In addition, some new information is obtained, such as the offender's personal information, crime, health and family background. These associated datasets imply some valuable information. But they are acquired from measurements or from human experts, with uncertain and noisy information. So it is still an important challenge to analyze these datasets from practical and theoretical perspectives [8], [9], [10]. In this paper, we present an analytical framework analyze combination factors for violent crime and introduce a fuzzy swarm rough set approach to extract the rules from the multidimension factor datasets.

\section{ANALYTICAL FRAMEWORK}

In our violent behavior analysis, there are seven main steps: data acquisition, original datasets, data preprocessing, result analysis, normalizing datasets, data analysis, rules and conclusions. In the data acquisition step, we obtain three kinds of data attributes including the environment data, psychology data and genotype data. All the data are collected into the original datasets. The detailed attributes will be presented in Section V. There are some duplicates, deletion, dispersion and manifest fault, etc in the original data. We have to preprocess these data by cleaning, transforming, integration and fltering and then all the "clean" and "integrated" data are stored as normalized datasets. We statistically analyze the dataset. Their information entropy is measured. Then the proposed fuzzy rough set reduction is introduced to reduce the dataset. The reduction results are analyzed locally (single attribute or combination of several attributes) and globally ( all attributes in one record or all records). The last step produces the output of some rules and conclusions. In summary, the complete system architecture is illustrated in Figure 1.

\section{Rough Set Reduction}

The basic concepts of rough set theory and its philosophy are presented and illustrated with examples in [11], [12], [13], [14], [15], [16]. Here, we illustrate only the relevant basic ideas of rough sets that are relevant to the present work.

In rough set theory, an information system is denoted in 4-tuple by $S=(U, A, V, f)$, where $U$ is the uni- 


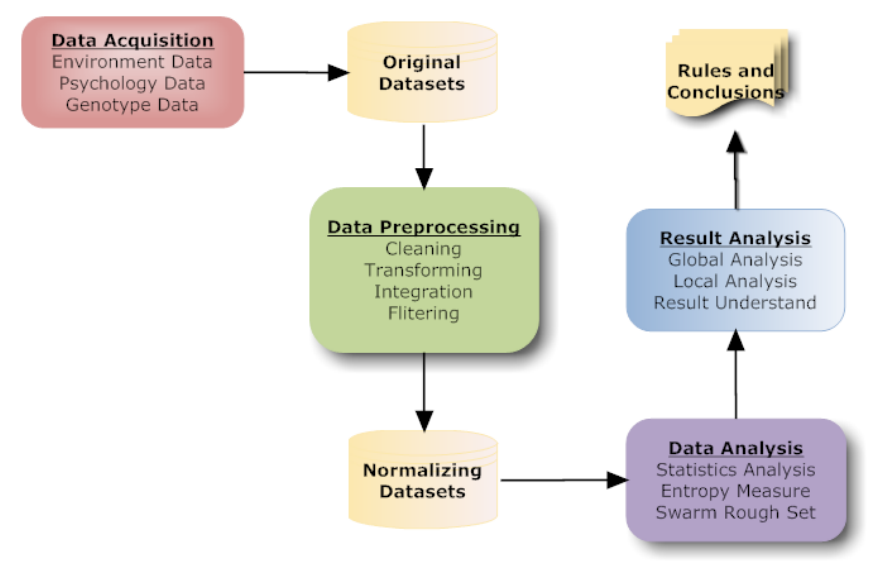

Figure 1. Overview of our architecture.

verse of discourse, a non-empty finite set of $N$ objects $\left\{x_{1}, x_{2}, \cdots, x_{N}\right\} . A$ is a non-empty finite set of attributes such that $a: U \rightarrow V_{a}$ for every $a \in A$ ( $V_{a}$ is the value set of the attribute $a$ ).

$$
V=\bigcup_{a \in A} V_{a}
$$

$f: U \times A \rightarrow V$ is the total decision function (also called the information function) such that $f(x, a) \in V_{a}$ for every $a \in A, x \in U$. The information system can also be defined as a decision table by $T=(U, C, D, V, f)$. For the decision table, $C$ and $D$ are two subsets of attributes. $A=\{C \cup D\}$, $C \cap D=\emptyset$, where $C$ is the set of input features and $D$ is the set of class indices. They are also called condition and decision attributes, respectively.

Definition 1: [Reduct] Given a decision table $T=$ $(U, C, D, V, f)$. The attribute $a \in B \subseteq C$ is $D-$ dispensable in $B$, if $P O S_{B}(D)=P O S_{(B-\{a\})}(D)$; otherwise the attribute $a$ is $D$-indispensable in $B$. If all attributes $a \in B$ are $D-$ indispensable in $B$, then $B$ will be called $D-$ independent. A subset of attributes $B \subseteq C$ is a $D$ - reduct of $C$, iff $P O S_{B}(D)=P O S_{C}(D)$ and $B$ is $D$-independent.

Definition 2: [Multi-reduct] Let $2^{|A|}$ represent all possible attribute subsets $\left\{\left\{a_{1}\right\}, \cdots,\left\{a_{|A|}\right\},\left\{a_{1}, a_{2}\right\}, \cdots,\left\{a_{1}, \cdots, \quad a_{|A|}\right\}\right\} . \quad$ Let $R E D$ represent the set of reducts, i.e.,

$$
\begin{aligned}
R E D=\{B \mid & P O S_{B}(D)=P O S_{C}(D), \\
& \left.\operatorname{POS}_{(B-\{a\})}(D)<\operatorname{POS}_{B}(D)\right\}
\end{aligned}
$$

Definition 3: [Multi-knowledge] Let $R E D$ represent the set of reducts, and $\varphi$ is a mapping from the condition space to the decision space. Then multi-knowledge can be defined as follows:

$$
\Psi=\left\{\varphi_{B} \mid B \in R E D\right\}
$$

Definition 4: [Reduced positive universe] Given a decision table $T=(U, C, D, V, f)$. Let $U / C=$ $\left\{\left[u_{1}^{\prime}\right]_{C},\left[u_{2}^{\prime}\right]_{C}, \cdots,\left[u_{m}^{\prime}\right]_{C}\right\}$, reduced universe $U^{\prime}$ can be written as:

$$
U^{\prime}=\left\{u_{1}^{\prime}, u_{2}^{\prime}, \cdots, u_{m}^{\prime}\right\} .
$$

And reduced positive universe $U_{\text {pos }}^{\prime}$ can be determined:

$$
U_{\text {pos }}^{\prime}=\left\{u_{i_{1}}^{\prime}, u_{i_{2}}^{\prime}, \cdots, u_{i_{t}}^{\prime}\right\} .
$$

Definition 5: [Reduced positive region] Given a decision table $T=(U, C, D, V, f)$. Let $\operatorname{POS}_{C}(D)=\left[u_{i_{1}}^{\prime}\right]_{C} \cup$ $\left[u_{i_{2}}^{\prime}\right]_{C} \cup \cdots \cup\left[u_{i_{t}}^{\prime}\right]_{C}$, where $\forall u_{i_{s}}^{\prime} \in U^{\prime}$ and $\left|\left[u_{i_{s}}^{\prime}\right]_{C} / D\right|=$ $1(s=1,2, \cdots, t) . \forall B \subseteq C$, reduced positive region

$$
\operatorname{POS}_{B}^{\prime}(D)=\bigcup_{X \in U^{\prime} / B \wedge X \subseteq U_{\text {pos }}^{\prime} \wedge|X / D|=1} X
$$

where $|X / D|$ represents the cardinality of the set $X / D$.

Consider Definitions 4 and 5, $\forall B \subseteq C, P O S_{B}(D)=$ $P O S_{C}(D)$ if $P O S_{B}^{\prime}=U_{\text {pos }}^{\prime}$ [16]. It is to be noted that $U^{\prime}$ is the reduced universe, which usually would reduce the scale of datasets significantly. It provides a more efficient strategy to observe the change of positive region when we search the reducts. We do not have to calculate $U / C, U / D$, $U / B, P O S_{C}(D), P O S_{B}(D)$ to compare $P O S_{B}(D)$ with $P O S_{C}(D)$ to determine whether they are equal to each other or not. We only calculate $U / C, U^{\prime}, U_{\text {pos }}^{\prime}, P O S_{B}^{\prime}$ and then compare $P O S_{B}^{\prime}$ with $U_{\text {pos }}^{\prime}$.

\section{Fuzzy Swarm Rough Set Reduction}

As mentioned above, finding all the reducts of information systems or decision tables is NP-complete problem [17], [18]. In this section, we present a fuzzy swarm algorithm for rough set reduction.

\section{A. Canonical swarm optimization}

The canonical swarm model consists of a swarm of individuals, which are initialized with a population of random candidate solutions. They move iteratively through the $d$ dimension problem space to search the new solutions, where the fitness, $f$, can be calculated as the certain qualities measure.

Each individual has a position represented by a positionvector $\vec{p}_{i}$ ( $i$ is the index of the individual), and a velocity represented by a velocity-vector $\vec{v}_{i}$. Each individual remembers its own best position so far in a vector $\vec{p}_{i}^{\#}$, and its $j$-th dimensional value is $p_{i j}^{\#}$. The best position-vector among the swarm so far is then stored in a vector $\vec{p}^{*}$, and its $j$ th dimensional value is $p_{j}^{*}$. During the iteration time $t$, the update of the velocity from the previous velocity to the new velocity is determined by Equ. (6). The new position is then determined by the sum of the previous position and the new velocity by Equ. (7).

$$
\begin{aligned}
v_{i j}(t)= & w v_{i j}(t-1)+c_{1} r_{1}\left(p_{i j}^{\#}(t-1)-p_{i j}(t-1)\right) \\
& +c_{2} r_{2}\left(p_{j}^{*}(t-1)-p_{i j}(t-1)\right)
\end{aligned}
$$




$$
p_{i j}(t)=p_{i j}(t-1)+v_{i j}(t)
$$

where $w$ is called as the inertia factor, $r_{1}$ and $r_{2}$ are the random numbers, which are used to maintain the diversity of the population, and are uniformly distributed in the interval $[0,1]$ for the $j$-th dimension of the $i$-th individual. $c_{1}$ is a positive constant, called as coefficient of the self-recognition component, $c_{2}$ is a positive constant, called as coefficient of the social component.

\section{B. Fuzzy swarm for rough set reduction}

Given a decision table $T=(U, C, D, V, f)$, the set of condition attributes, $C$, consist of $m$ attributes. We set up a search space of $m$ dimension for the rough set reduction.

Accordingly, each individual's position is represented as a vector with $m$ dimension. Each dimension of the individual's position maps one condition attribute. The individual's position is a series of priority levels of the attributes. The sequence of the attribute will not be changed during the iteration. The domain for each dimension is limited from 0 to 1 . The value ' 1 ' means the corresponding attribute is selected definitely while ' 0 ' not selected definitely. Otherwise the attribute would be selected with the probability according to the value of the individual's position. So each position can be "decoded" to a potential reduction solution, an subset of $C$. In other words, we can map $C$ into a fuzzy set $R$ using a fuzzy reduct relation through the individual's fuzzy positionvector. The fuzzy reduct relation $\vec{p}_{i}$ between $C$ and $R$ has the following meaning: for each element in the position-vector $\vec{p}_{i}$ ( $i$ is the index of the individual), the element

$$
p_{i j}=\mu_{F}\left(C_{j}, R_{j}\right)
$$

where $\mu_{F}$ is the membership function, $i \in\{1,2, \cdots, n\}, j \in$ $\{1,2, \cdots, m\}$. In the attribute reduction problem, the elements of the solution must satisfy the following conditions:

$$
p_{i j} \in[0,1], i \in\{1,2, \cdots, n\}, j \in\{1,2, \cdots, m\}
$$

Here $p_{i j}$ represents the degree of membership of the $i$-th element $c_{i}$ in domain $C$ and the $j$-th element $r_{j}$ in domain $R$ to relation $\vec{p}_{i}$.

In the crisp mode, the individual's position in the considered dimension is positive, the corresponding attribute is selected definitely. And the negative direction implies that it is not selected definitely. In the fuzzy mode, the individual's position is fuzzified, which is propagated from its velocity. At each time iterating step, each individual updates its velocity according to Equ. (6). And the position is updated according to Equ. (7). In our fuzzy swarm model, the position is fuzzified by the sigmoid function:

$$
\Gamma\left(p_{i j}(t)\right)=\frac{1}{1+e^{-p_{i j}(t)}}
$$

Figure 2 illustrates how the position is reacted on by its velocity when the individuals' maximum positions output

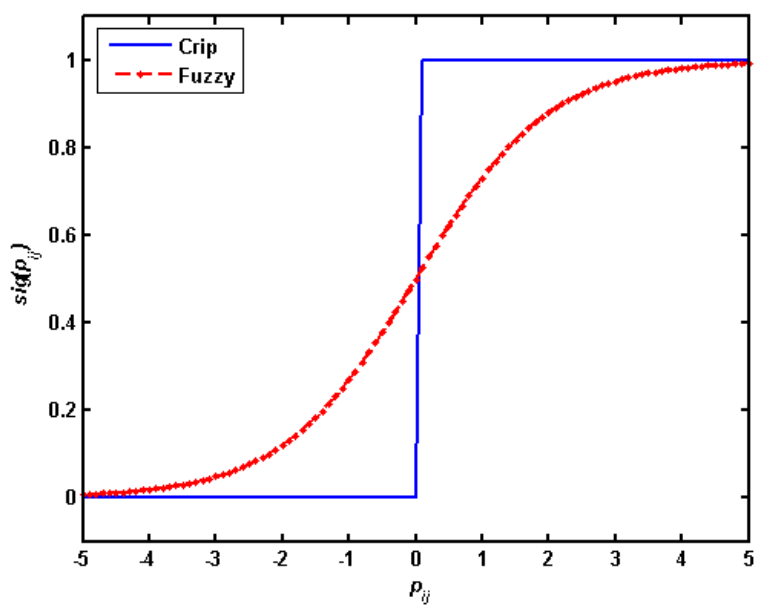

Figure 2. Curve of membership functions.

from Equ. (7) are clamped in between $[-5,5]$. The membership functions are similar with the ones in [19].

Since the position indicates the potential reduction solution, we should "decode" the fuzzy vectors and get the feasible solutions. The position values are compared with a random number $\rho$ in the interval $[0,1]$. If the position value is larger than $\rho$, the position would be defuzzied as ' 1 ', i.e. the corresponding selection flag $s_{i j}(t)$ is set to ' 1 '. And the corresponding attribute with the position is selected. Otherwise the attribute would be not selected as Equ. (11). After all the elements of the position vector have been processed, we get the reduction solution from the fuzzy swarm model.

$$
s_{i j}(t)= \begin{cases}1 & \text { if } \Gamma\left(p_{i j}(t)\right)>\rho \\ 0 & \text { otherwise }\end{cases}
$$

If it is a feasible solution, we calculate the attribute number in the subset of attributes. The solution with the lowest number would be selected. For the swarm, the lower number of attributes in the feasible solution, the better the fitness of the corresponding individual is. So the individual's fitness is determined by Equ. (12). If the reduced universe of discourse is non-equal to the reduced positive region, i.e. $P O S_{E}^{\prime} \neq U_{p o s}^{\prime}$, the fitness is punished as the total number of the condition attributes, otherwise the fitness is the attribute number of the potential reduction solution represented by the individual's position.

$$
f_{D}(E)= \begin{cases}|E| & \text { if } \operatorname{POS}_{E}^{\prime}(D)=U_{\text {pos }}^{\prime} \\ |C| & \text { if } \operatorname{POS}_{E}^{\prime}(D) \neq U_{\text {pos }}^{\prime}\end{cases}
$$




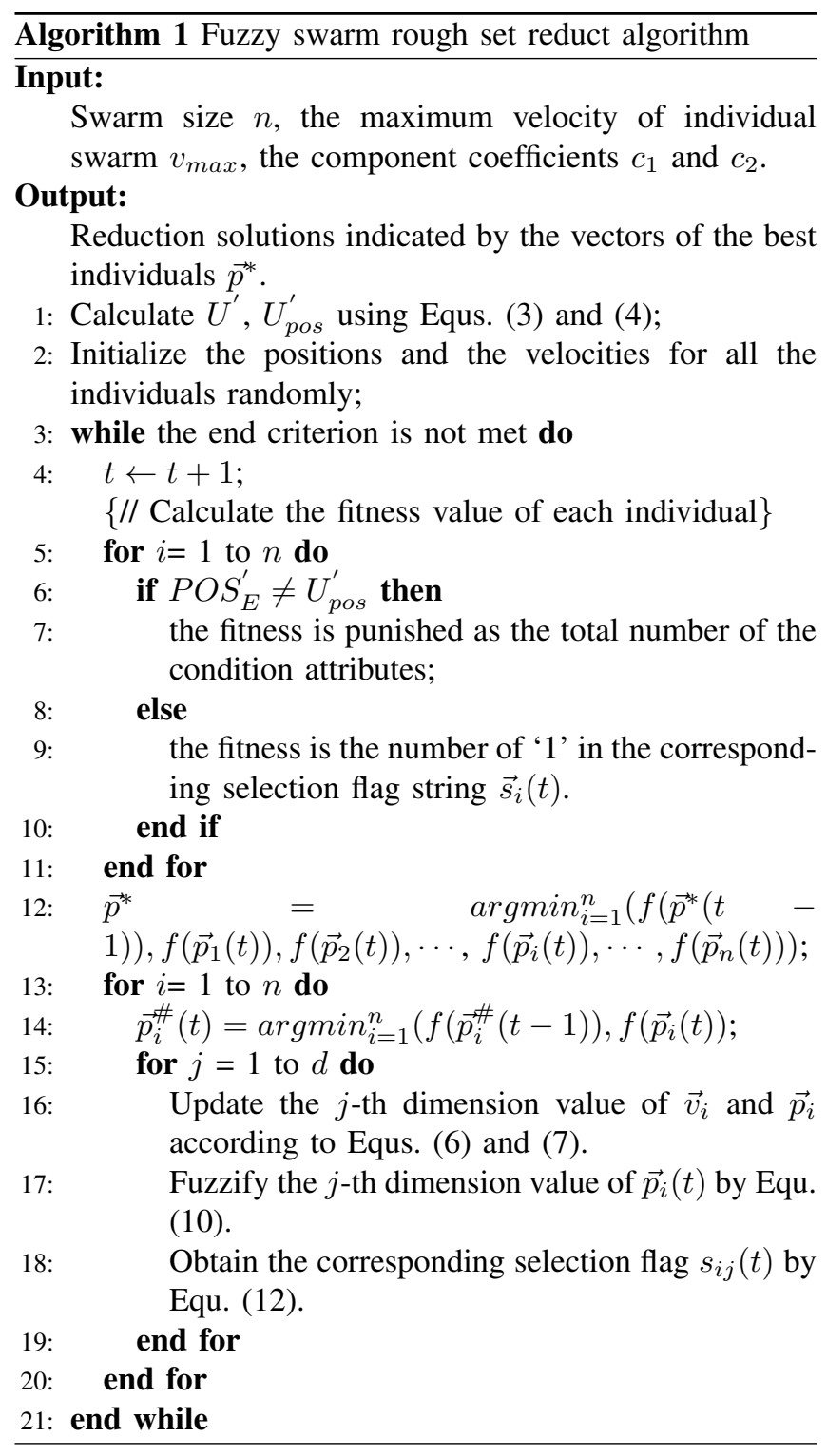

\section{EXPERIMENTS AND RESULTS}

\section{A. Dataset acquiring}

The dataset is acquired by the Chongqing Municipal Public Security Bureau, Institute of Environmental Systems Biology and School of Information Science and Technology of Dalian Maritime University. All informed consents were obtained before participation. During the acquiring process of dataset, the considered factors include the family environment (degree of education, occupation, and family economic status), $\mathrm{P}$ and $\mathrm{N}$ psychological factor, MAOA and DRD4 genotypes, up to 64 factors. The family environment information is acquired by participant's information questionnaire. The psychological information is obtained by Eysenck personality questionnaire. Genetic information is obtained by polymerase chain reaction (PCR).
Table II

PARAMETER SETTINGS FOR THE ALGORITHMS.

\begin{tabular}{lll}
\hline Algorithm & Parameter name & Value \\
\hline \multirow{4}{*}{ DR } & $\begin{array}{l}\text { Number of sampling levels } \\
\text { Number of subtables }\end{array}$ & 5 \\
$\quad$ to sample per level & 10 \\
& $\begin{array}{l}\text { Smallest subtable size } \\
\quad \text { (lowest level) }\end{array}$ & $50 \%$ \\
& Largest substable size & $90 \%$ \\
$\quad \quad$ (highest level) & \\
& Swarm size & $($ even $)(\operatorname{int})(10+2 * \operatorname{sqrt}(L))$ \\
Swarm & Self coefficient $c_{1}$ & $0.5+\log (2)$ \\
& Social coefficient $c_{2}$ & $0.5+\log (2)$ \\
& Inertia weight $w$ & 0.91 \\
& Clamping Coefficient $\phi$ & 0.5 \\
\hline
\end{tabular}

In the process of data acquisition, there are some missing, redundant and even data with some tolerances. There are three processing methods for handling these type of data records: no processing, filling or deleting. After cleaning, transforming, integration and filtering, the datasets are original 67 attributes (columns) and 2486 records (rows) to normalized 20 attributes (columns) and 578 records (rows) as illustrated in Table I.

\section{B. Rough set extraction}

After the normalized datasets are discretized, we make an attempt to reduce the datasets of combination factors using the proposed fuzzy swarm rough set algorithm. In our experiments, the algorithm used for comparison was dynamic reducts (DR) [20], [21]. Dynamic reducts (DR) is first defined by Bazan et al. [20], [21]. A number of subtables are randomly sampled from the input table, and proper reducts are computed from each of these using some algorithms. The reducts that occur the most across subtables are in some sense the "most stable" [22]. Both methods are valid and efficient in rough set reduction field due to their strong convergence properties. Our algorithms were implemented in the $\mathrm{C}++$ language and their parameters settings are chosen in accordance with the recommendations of Clerc [23] and Liu [24]. The computation environment was an Intel ${ }^{\circledR}$ Core $^{T M}$ Duo CPU T2250@1.73 GHz processor with 1G memory. Specific parameter settings for the algorithms are described in Table II, where $L$ is the length of condition attributes. Each experiment (for each algorithm) was repeated 10 times with different random seeds. The average fitness values of the best solutions throughout the optimization run were recorded. The average number of surplus property and the number of optimal solutions in 10 trials are shown in Figure 3 . The fuzzy swarm algorithm usually achieves better results than DR method. In addition, multiple fuzzy swarm usually produces multiple candidate reducts, allowing for the possibility of multi-knowledge extraction. 
Table I

DATASET CLEANING.

\begin{tabular}{|c|c|c|c|c|c|c|c|c|}
\hline \multirow[b]{2}{*}{ Item } & \multicolumn{4}{|c|}{ Before Preprocessing } & \multicolumn{4}{|c|}{ After Preprocessing } \\
\hline & Environment & Psychology & Genotype & $\overline{\text { Total }}$ & Family & Psychology & Genotype & Total \\
\hline Column & 63 & 2 & 2 & 67 & 16 & 2 & 2 & 20 \\
\hline Record & 1233 & 1233 & 2486 & 2486 & 578 & 578 & 578 & 578 \\
\hline
\end{tabular}

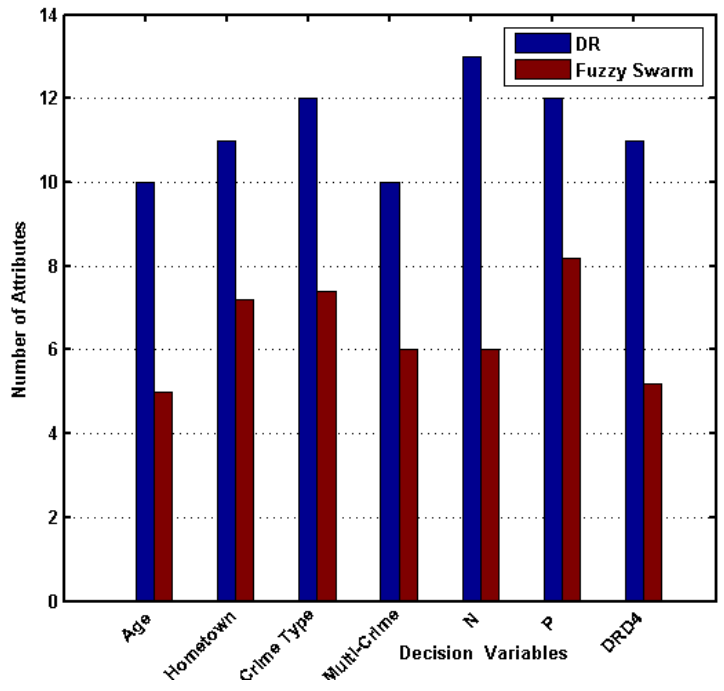

Figure 3. Number of attributes depending on decision variables.

According to the experimental data and results, the following rules are extracted:

1) The characteristics of the young-offenders are as follows, especially these from the single-parent family: economic condition is in the low middle level; EPQ$\mathrm{P}$ score is high; junior high school educational level; DRD4 is middle-sized and MAOA is the third type or the fourth. The confidence is $79 \%$.

2) The characteristics of the minority nationality are as follows: education level is low; unemployed; economic condition is poor and EPQ-P is the middle score or the high. The confidence is $72 \%$.

3) The characteristics of the adult-offenders from east of Chongqing are as follows: EPQ-N score is middle or high and most of their educational level is highlyeducated. The confidence is $68 \%$.

4) The characteristics of the prisoners are as follows: DRD4 is abnormal; EPQ-P and EPQ-N scores are middle or high; MAOA is the fifth type and economic condition is the lower middle level and some are unemployed. The confidence is $65 \%$.

5) The characteristics of the prisoners are as follows: they are bibulous; EPQ-P score is middle or high; economic condition is in the lower middle level; term of penalty is longer and DRD4 is abnormal, most of them are from east of Chongqing. The confidence is $70 \%$.

6) The characteristics of the adult-offenders are as follows: their childhood is abused; EPQ-P and EPQ-N scores are middle or high; economic condition is in the lower middle level and some are unemployed; term of penalty is longer and educational level is junior high school. The confidence is $71 \%$.

These results are helpful to further understand the factors of violent crime.

\section{CONCLUSIONS AND FURTHER WORKS}

This paper presented a novel fuzzy swarm rough set to analyze three kinds of combination factors, i.e. psychological, environmental and genetic factors. We evaluated the performance of our approach using the violent factor datasets and the corresponding computational experiments. Empirical results indicate that our approach is ideal for all the considered problems and the fuzzy swarm optimization technique outperformed the dynamic reducts (DR) approach by obtaining multiple reductions for the combination factor datasets. Although some knowledges should be analyzed and verified the correctness by neuroscientists further, the approach is helpful for violent behavior analysis. More methods [25-27] and some detailed underlying mechanism should be investigated further.

\section{ACKNOWLEDGMENT}

The authors would like to thank Jian Jiao, Bo Liu, Shui Zhou, Meng Zhang, Yu Feng and Ying Fu for their scientific collaboration in this research work. This work is supported partly by the Nature Science Foundation of Chongqing of China (Grant No. 2009BA5074), the National Natural Science Foundation of China (Grant No. 61173035), the Fundamental Research Funds for the Central Universities (Grant No. 2012TD027), the Program for New Century Excellent Talents in University (Grant No. NCET-11-0861), and the Dalian Science and Technology Fund (Grant No. 2010J21DW006).

\section{REFERENCES}

[1] E. Teixeira and P. Dalgalarrondo, "Violent crime and dimensions of delusion: a comparative study of criminal and noncriminal delusional patients," Journal of the American 
Academy of Psychiatry and the Law Online, vol. 35, no. 2, pp. 225-231, 2009.

[2] R. Cadoret, W. Yates, and E. Troughton, "Gene-environment interaction in the genesis of aggressivity and conduct disorders," Archives of General Psychiatry, vol. 52, no. 11, pp. 916-924, 1995.

[3] F. Yen, C. Hong, S. Hou, J. Wang, and S. Tsai, "Association study of serotonin transporter gene vntr polymorphism and mood disorders, onset age and suicide attempts in a chinese sample," Neuropsychobiology, vol. 48, no. 1, pp. 5-9, 2003.

[4] F. Ducci, T. Newman, S. Funt, G. Brown, M. Virkkunen, and D. Goldman, "A functional polymorphism in the maoa gene promoter (maoa-lpr) predicts central dopamine function and body mass index," Molecular Psychiatry, vol. 11, no. 9, pp. 858-866, 2006.

[5] M. Rutter, "Biological implications of gene-environment interaction," Journal of Abnormal Child Psychology, vol. 36, no. 7, pp. 969-975, 2008.

[6] H. J. Eysenck and S. Eysenck, "Manual of the eysenck personality questionnaire (junior and adult)," 1975.

[7] J. Philippe Rushton, D. Fulker, M. Neale, D. Nias, and H. Eysenck, "Ageing and the relation of aggression, altruism and assertiveness scales to the eysenck personality questionnaire," Personality and Individual Differences, vol. 10, no. 2, pp. 261-263, 1989.

[8] K. Taipale, "Data mining and domestic security: connecting the dots to make sense of data," Columbia Science and Technology Law Review, vol. 5, p. 2, 2003.

[9] H. Chen, W. Chung, J. Xu, G. Wang, Y. Qin, and M. Chau, "Crime data mining: a general framework and some examples," Computer, vol. 37, no. 4, pp. 50-56, 2004.

[10] P. Phillips and I. Lee, "Mining co-distribution patterns for large crime datasets," Expert Systems with Applications, vol. 39, no. 14, p. 11556C11563, 2012.

[11] Z. Pawlak, "Rough sets," International Journal of Computer and Information Sciences, vol. 11, no. 1-2, pp. 341-356, 1982.

[12] — , "Rough sets and intelligent data analysis," Information Sciences, vol. 147, no. 1-4, pp. 1-12, 2002.

[13] Q. Wu and D. Bell, "Multi-knowledge extraction and application," Rough Sets, Fuzzy Sets, Data Mining, and Granular Computing, Lecture Notes in Computer Science, vol. 2639, pp. 574-575, 2003.

[14] N. Zhong, J. Dong, and S. Ohsuga, "Using rough sets with heuristics for feature selection," Journal of Intelligent Information Systems, vol. 16, no. 3, pp. 199-214, 2001.

[15] G. Wang, "Rough reduction in algebra view and information view," International Journal of Intelligent Systems, vol. 18, no. 6, pp. 679-688, 2003.
[16] Z. Xu, Z. Liu, B. Yang, and W. Song, "A quick attribute reduction algorithm with complexity of $\max$ $\left(O(|C||U|), O\left(|C|^{2}|U / C|\right)\right)$," Chinese Journal of Computers, vol. 29, no. 3, pp. 391-399, 2006.

[17] M. Garey and D. Johnson, Computers and Intractability: A Guide to the Theory of NP-completeness. WH Freeman \& Co., 1979.

[18] Q. Hu, X. Li, and D. Yu, "Analysis on classification performance of rough set based reducts," in Proceedings of the 9th Pacific Rim International Conference on Artificial Intelligence, PRICAI 2006: Trends in Artificial Intelligence, Lecture Notes in Artificial Intelligence, v4099. Springer, 2006, pp. 423-433.

[19] Q. Hu, W. Pan, L. Zhang, D. Zhang, Y. Song, M. Guo, and D. Yu, "Feature selection for monotonic classification," IEEE Transactions on Fuzzy Systems, vol. 20, no. 1, pp. 69-81, 2012.

[20] J. G. Bazan, A. Skowron, and P. Synak, "Dynamic reducts as a tool for extracting laws from decisions tables," in Proceedings of International Symposium on Methodologies for Intelligent Systems, Lecture Notes in Artificial Intelligence, vol. 869. Springer, 1994, pp. 346-355.

[21] J. Bazan, "A comparison of dynamic and non-dynamic rough set methods for extracting laws from decision tables," Rough Sets in Knowledge Discovery, vol. 1, pp. 321-365, 1998.

[22] A. Øhrn, "Discernibility and rough sets in medicine: Tools and applications," Ph.D. dissertation, Norwegian University of Science and Technology, 1999.

[23] M. Clerc and J. Kennedy, "The particle swarm-explosion, stability, and convergence in a multidimensional complex space," IEEE Transactions on Evolutionary Computation, vol. 6, no. 1, pp. 58-73, Feb. 2002.

[24] H. Liu, A. Abraham, and M. Clerc, "Chaotic dynamic characteristics in swarm intelligence," Applied Soft Computing, vol. 7, no. 3, pp. 1019-1026, 2007.

[25] H. Liu, A. Abraham, A.E. Hassanien, Scheduling jobs on computational grids using a fuzzy particle swarm optimization algorithm, Future Generation Computer Systems, 26 (8), pp. 1336-1343, 2010.

[26] A Abraham, Intelligent systems: Architectures and perspectives, Recent advances in intelligent paradigms and applications, pp. 1-35, 2003.

[27] F Xhafa, E Alba, B Dorronsoro, B Duran, A Abraham, Efficient batch job scheduling in grids using cellular memetic algorithms, Metaheuristics for Scheduling in Distributed Computing Environments, pp. 273-299, 2008. 